\title{
Duopoly Models for Power Trading in Secure Cooperative Relay Networks
}

\author{
Khyati Chopra \\ Dept. of Electrical Engineering \\ Indian Institute of Technology, Delhi \\ New Delhi-110016, India \\ Email: eez148071@ee.iitd.ac.in
}

\author{
Ranjan Bose \\ Dept. of Electrical Engineering \\ Indian Institute of Technology, Delhi \\ New Delhi-110016, India \\ Email: rbose@ee.iitd.ac.in
}

\author{
Anupam Joshi \\ Dept. of Computer Science \\ University of Maryland, Baltimore County \\ Baltimore, MD 21250, United States \\ Email: joshi@cs.umbc.edu
}

\begin{abstract}
In this paper we consider a source, in-need of cooperation of a relay due to limited battery power to communicate with the destination, with an eavesdropper tapping the second hop, when all the links undergo Rayleigh fading. Relay nodes are the market players, who compete to trade their power to source in order to maximize their incentives. Asymptotic analysis of the intercept probability is presented when source-relay and relay-destination average SNRs are the same or different. We evaluate the utility of relays in various novel duopoly economic communication models and show that increase in channel gain from relay to eavesdropper, decreases the profit of relays. We also demonstrate that the choice of strategic variable as output or price, can have a dramatic effect on the Nash outcome in an oligopoly interactive market.
\end{abstract}

\section{INTRODUCTION}

In the communication network, cooperative relays play a significant role to save the uplink transmit power of mobile terminals [1] and improve involved network performances, especially in the areas where signal strength is poor due to geographic referenced constraints [2]. These cooperative networks are susceptible to eavesdropping due to the broadcast nature of wireless medium and therefore information theoretic security is emerging as a paradigm approach to assure the secure communication by exploiting the physical characteristics of wireless channels [3]-[6]. Here, we consider such a cooperative and secure communication network in a competitive oligopoly market scenario as a means to trade power in mobile communication for local power efficiency and reliability.

Oligopoly market strategic theory deals with the competition between two or more firms as investigated in [7]-[9]. In [10] for dual-hop transmission, the fixed relay network is modeled as a virtual oligopoly market, and the Cournot game model is exploited to solve the resource allocation problem. Various price competition models for emerging mobile networks have been studied in [11]. Authors in [12] have presented a business model using sealed bid procurement auction based game theory for power-trading in cooperative networks. [13] investigated the profit of price for spectrum sharing in cognitive radio using genetic algorithm with supermodular game. In contrast to the above study, our work is motivated to exploit various

978-1-5090-2361-5/16/\$31.00 (c) 2016 IEEE pricing models of oligopoly market in a cooperative relay network for power trading, in presence of an eavesdropper. Cournot and Bertrand constitutes the two most classical models of the oligopoly market [7] , [8]. Under static Cournot model, the firms produce perfectly homogeneous goods and simultaneously choose output quantity knowing market demand and cost conditions whereas, in Bertrand model the firms compete by strategically managing their price. Firms enjoy positive profits in Cournot as the resulting market prices exceed that of the marginal costs while price equals marginal cost in Bertrand. The perfectly competitive solution is reached in the Bertrand model as long as there are two or more competitors [9]. In contrast, the Cournot solution approaches the perfectly competitive equilibrium only when number of firms approaches infinity. In a dynamic Cournot scenario, one firm produces output in the first period while the other firm produces output in the second period. Stackelberg identified the sub-game perfect Nash equilibrium (SPNE) for this model and examined how dynamic considerations can give first mover a strategic advantage over the other [7]. This can be extended to a dynamic Bertrand game, where one firm chooses price in the first period while the other chooses price in the second period, but on the contrary, here second mover earns higher profits.

In [14] the authors show that for Cournot-Bertrand model, where two firms have the choice to compete in output or in price, under certain demand and cost conditions the dominant strategy for each firm is to compete in output rather than price. In [15] the authors investigated the Cournot-Bertrand model when the degree of product differentiation is allowed to vary and examined that when the products are homogeneous, the perfectly competitive outcome results in which the Cournottype firm produces the competitive level of market output, while the Bertrand-type firm exits the market.

In this paper, we first evaluate the intercept probability for a dual hop cooperative threshold based decode-and-forward (DF) relaying network model in Section II. As is known, an intercept event occurs when the secrecy capacity becomes negative. Using the expression, we examine the market strategic game framework, where relay nodes use different duopoly models to trade their power to source in Section III. We also 
investigate how gains of the eavesdropper's link, affect the utility of relays in this market game, which in turn motivates relay to keep low intercept probability to earn high incentives. In Section IV comparative numerical analysis of different duopoly models is done and finally, we conclude this study in Section V.

\section{SySTEM MOdeL}

We consider a source $S$, two DF relays $R_{i}, i \in[1,2]$, a destination $D$ and an eavesdropper $E$ in the system. We assume there is no direct $S-D, S-E$ link and the communication takes place with the help of a single cooperative relay. Also, each node in the system model works in half-duplex mode with all the channels identically and independently distributed and the instantaneous channel state information (ICSI) of the main channel as well as of the eavesdropper channel is available. The $S-R_{i}$ channels $h_{s r_{i}}, R_{i}-D$ channels $h_{r_{i} d}$, and $R_{i}-E$ channels $h_{r_{i} e}, \forall i \in[1,2]$, are slowly varying Rayleigh flat faded channels [16]. Let $P_{s}$ and $P_{r_{i}}$ denote the average powers used at source and relay $R_{i}$ respectively. Also, let $N_{s r_{i}}, N_{r_{i} d}$ and $N_{r_{i} e}$ denote the variances of additive white Gaussian noise of $S-R_{i}, R_{i}-D$, and $R_{i}-E$ links respectively. The SNRs $\Gamma_{s r_{i}}, \Gamma_{r_{i} d}$ and $\Gamma_{r_{i} e}$ are exponentially distributed given as $\Gamma_{s r_{i}}=\frac{P_{s}\left|h_{s r_{i}}\right|^{2}}{N_{s r_{i}}}, \Gamma_{r_{i} d}=\frac{P_{r_{i}}\left|h_{r_{i} d}\right|^{2}}{N_{r_{i}} d}, \Gamma_{r_{i} e}=\frac{P_{r_{i}} \mid h_{r_{i} e} e^{2}}{N_{r_{i} e}}$ with average values $1 / \beta_{s r_{i}}, 1 / \beta_{r_{i} d}$ and $1 / \alpha_{r_{i} e}$ respectively, where $\beta_{s r_{i}}, \beta_{r_{i} d}$ and $\alpha_{r_{i} e}$ are the parameters of the exponential distribution.

Deviating from the fact of perfect decoding at all the relays, [17] considers only a set of relays can successfully decode the message among all the relays. Let us consider $\gamma_{t h}$ as the predetermined threshold value for $S-R_{i}$ link, and relays can decode the message only if the SNR of $S-R_{i}$ link, $\Gamma_{s r_{i}}$ is greater than $\gamma_{t h}$ else, no communication takes place as there is no direct link between $S-D$ or $S-E$. The secrecy capacity is shown to be the difference between the capacity of main link and that of wiretap link in [3] , [18]

$$
C_{s} \triangleq \frac{1}{2}\left[\log _{2}\left(\frac{1+\Gamma_{M}}{1+\Gamma_{E}}\right)\right]^{+}
$$

where $\Gamma_{M}=\frac{P_{r_{i}}\left|h_{r_{i} d}\right|^{2}}{N_{r_{i} d}}$ is the SNR of the main link and $\Gamma_{E}=\frac{P_{r_{i}}\left|h_{r_{i} e}\right|^{2}}{N_{r_{i} e}}$ is the SNR of the eavesdropper link.The term $1 / 2$ here denotes that two time slots are required to complete this dual hop transmission process. An intercept event occurs when the secrecy capacity is negative and probability that the eavesdropper successfully intercepts the source signal is called intercept probability $P_{i n t}$, [16] which is a key metric in evaluating the performance of physical-layer security. Using (1), we evaluate intercept probability for single $i^{\text {th }}$ relay as

$$
\begin{aligned}
P_{i n t}^{i} & =\mathbb{P}\left[\Gamma_{s r_{i}} \geq \gamma_{t h}\right] \mathbb{P}\left[C_{s} \leq 0\right]+\mathbb{P}\left[\Gamma_{s r_{i}} \leq \gamma_{t h}\right] \\
& =\mathbb{P}\left[\frac{P_{s}\left|h_{s r_{i}}\right|^{2}}{N_{s r_{i}}} \geq \gamma_{t h}\right] \mathbb{P}\left[\frac{1}{2}\left[\log _{2}\left(\frac{1+\Gamma_{r_{i} d}}{1+\Gamma_{r_{i} e}}\right)\right] \leq 0\right] \\
& +\mathbb{P}\left[\frac{P_{s}\left|h_{s r_{i}}\right|^{2}}{N_{s r_{i}}} \leq \gamma_{t h}\right]
\end{aligned}
$$

$$
\begin{aligned}
& =\left[1-\mathbb{P}\left[\frac{P_{s}\left|h_{s r_{i}}\right|^{2}}{N_{s r_{i}}} \leq \gamma_{t h}\right]\right] \mathbb{P}\left[\frac{1+\Gamma_{r_{i} d}}{1+\Gamma_{r_{i} e}} \leq 1\right] \\
& +\left(1-e^{-\gamma_{t h} \beta_{s r_{i}}}\right) \\
& =\left(1-\left(1-e^{-\gamma_{t h} \beta_{s r_{i}}}\right)\right) \mathbb{P}\left[\Gamma_{r_{i} d} \leq \Gamma_{r_{i} e}\right]+\left(1-e^{-\gamma_{t h} \beta_{s r_{i}}}\right) \\
& =\frac{e^{-\gamma_{t h} \beta_{s r_{i}}} \beta_{r_{i} d}}{\beta_{r_{i} d}+\alpha_{r_{i} e}}+1-e^{-\gamma_{t h} \beta_{s r_{i}}}
\end{aligned}
$$

It is evident from (2) that probability of positive secrecy capacity depends upon the statistics of the $R_{i}-D$ and $R_{i}-E$ links only. Secondly, since $S-R_{i}$ link is common, the instantaneous secrecy capacity is negative only when $\Gamma_{r_{i} d} \leq \Gamma_{r_{i} e}$, and hence it is same as that of wiretap channel with relay as a source. This $P_{i n t}^{i}$ plays an important role for relay nodes to improve their incentives in this oligopoly market strategic game.

\section{DuOpoly Game Formulation}

This section deals with various novel duopoly economic models for dual hop cooperative relay network in presence of an eavesdropper. The utility of the relay node will be affected by the intercept probability and also by the choice of strategic variable as output or price under static or dynamic conditions. In all schemes, these relay nodes are closely related in terms of their location and channel statistics. Therefore, as intercept probability depends on the channel statistics of the relay nodes which are nearly same, $P_{i n t}^{1}=P_{i n t}^{2}=P_{i n t}$ is considered and homogeneity is maintained in this market game.

\section{A. Cournot-Cournot (CC) Model}

In the $\mathrm{CC}$ model, both the competitive relay nodes choose quantity as their strategic variable simultaneously, under the assumption of complete information, linear demand function and fixed cost per unit resource. The quantity $Q_{i}$ produced by the two relay nodes, is taken as the quantity of their powers resources $P_{r_{1}}$ and $P_{r_{2}}$, such that $Q_{1}=P_{r_{1}}$ and $Q_{2}=P_{r_{2}}$ respectively. Due to homogeneity, the product differentiation parameter $d$ is considered as one [15]. When $d=0$, each relay is an independent monopolist. Let the price per unit power resource for the $i^{t h}$ relay be $P_{i}$

$$
\begin{aligned}
& P_{1}=A\left(1-P_{i n t}\right)-Q_{1}-d Q_{2} \\
& P_{2}=A\left(1-P_{i n t}\right)-Q_{2}-d Q_{1}
\end{aligned}
$$

where $d=1$ due to homogeneity

$$
P_{i}=A\left(1-P_{i n t}\right)-Q
$$

where $Q=\sum_{i=1}^{2} Q_{i}$, is the total quantity or the total market demand. This is an inverse demand function [9], where the price set by the relays will decrease with the increase in the demand of power resource by the source node. Also, it varies inversely with intercept probability, which in turn motivates the relay nodes to maintain high secrecy capacity in order to maximize their incentives. $A$ is the positive constant. Let the marginal cost $C$ be same for both relays, and $C_{i}=C Q_{i}$, where $C_{i}$ is the total cost of power production. The utility function of the $i^{t h}$ relay $\pi_{i}$ is given as the difference between 
the total revenue and the total cost

$$
\begin{aligned}
& \pi_{1}=\left(A\left(1-P_{i n t}\right)-\left(Q_{1}+Q_{2}\right)\right) Q_{1}-C Q_{1} \\
& \pi_{2}=\left(A\left(1-P_{i n t}\right)-\left(Q_{2}+Q_{1}\right)\right) Q_{2}-C Q_{2}
\end{aligned}
$$

The second-order condition of profit maximization holds, because the second derivative of the profit function for each relay is -2 which is negative hence, best response $Q_{i}^{*}$ of $i^{t h}$ relay using (6) and (7) is

$$
\begin{aligned}
Q_{1}^{*} & =\frac{A\left(1-P_{i n t}\right)-C-Q_{2}}{2} \\
Q_{2}^{*} & =\frac{A\left(1-P_{i n t}\right)-C-Q_{1}}{2}
\end{aligned}
$$

At Nash equilibrium both the best responses $B R_{i}$ intersect

$$
\begin{aligned}
& Q_{1}^{*}=B R_{1}\left(Q_{2}^{*}\right) \\
& Q_{2}^{*}=B R_{2}\left(Q_{1}^{*}\right)
\end{aligned}
$$

After substituting both equations, we get the Cournot Nash equilibrium solution as

$$
\begin{aligned}
\left(Q_{1}^{*}, Q_{2}^{*}\right) & =\left(\frac{A\left(1-P_{i n t}\right)-C}{3}, \frac{A\left(1-P_{i n t}\right)-C}{3}\right) \\
\left(\pi_{1}^{*}, \pi_{2}^{*}\right) & =\left(\frac{\left(A\left(1-P_{i n t}\right)-C\right)^{2}}{9}, \frac{\left(A\left(1-P_{i n t}\right)-C\right)^{2}}{9}\right)
\end{aligned}
$$

As there is no product differentiation and cost functions are same for both the relays, homogeneity and symmetricity is intuitively maintained in this model.

\section{B. Bertrand-Bertrand (BB) Model}

In the BB model, both the competitive relay nodes choose price as their strategic variable simultaneously, under the same demand and cost conditions as CC model. Solving for output, the demand function is $Q=A\left(1-P_{\text {int }}\right)-P_{i}$, where $Q$ is the total quantity demanded. Here, the relay's problem is to maximize with respect to $P_{i}$ instead of $Q_{i}$ [7]. Once relays set prices, consumers determine quantity demanded. As the products are homogeneous, source will always purchase from the cheapest seller. If prices are the same, $P=P_{1}=P_{2}$, source is indifferent between purchasing from relay 1 and 2 . In this case, the usual assumption is that the source demand is equally shared [9] among both the relays.

At Bertrand Nash equilibrium, price $P_{i}$ equals marginal cost $C$ hence, utility $\pi_{i}$ of both relays becomes zero and the perfectly competitive solution is reached. Under these conditions, the quantity demand faced by $i^{\text {th }}$ relay is

$$
Q_{i}^{*}=\frac{A\left(1-P_{\text {int }}\right)-C}{2}
$$

Here, duopoly results in perfect competition and is enough to push prices down to the marginal cost $C$ level, commonly referred as Bertrand paradox in economics literature [9].

\section{Cournot-Bertrand (CB) Model}

We analyze a Cournot-Bertrand model where one relay competes in output and the other competes in price. With general linear inverse demand functions and perfectly homogeneous products, we show that the unique Nash equilibrium is the perfectly competitive equilibrium, where price equals marginal cost $C$. Hence, the Bertrand-type relay exits the market and the Cournot-type relay produces the perfectly competitive level of market output [8]. We can also consider a differentiated duopoly game where relay 1 competes in output, $Q_{1}$ and relay 2 competes in price, $P_{2}$. Relay's profit function depends on both strategic variables, $\pi_{i}\left(Q_{1}, P_{2}\right)$, with a natural asymmetry in the model [15]. In a non-cooperative setting, the structure of the model guarantees a unique Nash equilibrium, where both the best-response functions intersect. We illustrate these results with linear demand and cost functions that are similar to those found in Singh and Vives [14]. Linear demand functions with different strategic choice variables is given in [15] as

$$
\begin{aligned}
P_{1} & =A\left(1-P_{\text {int }}\right)(1-d)-\left(1-d^{2}\right) Q_{1}+d P_{2} \\
Q_{2} & =A\left(1-P_{\text {int }}\right)-P_{2}-d Q_{1}
\end{aligned}
$$

where $A \in(0, \infty), d=1$ due to homogeneity and quantity $Q_{i}$ of the two relay nodes are their power resources $Q_{1}$ and $Q_{2}$ respectively. Costs of production is same for both relays, and $C$ equals marginal cost. Thus, the profit function for $i^{\text {th }}$ relay is $\pi_{i}=\left(P_{i}-C\right) Q_{i}, \forall i=1,2$. Here, relays face symmetric cost functions and differ only in their choice of strategic variable. The Nash equilibrium values in the static Cournot-Bertrand model, when the best response functions intersect are given as

$$
\begin{aligned}
& P_{1}^{*}=\frac{A\left(1-P_{i n t}\right)\left(2-d-2 d^{2}+d^{3}\right)+C(2+d-}{4-3 d^{2}} \\
& P_{2}^{*}=\frac{A\left(1-P_{i n t}\right)\left(2-d-d^{2}\right)+C\left(2+d-2 d^{2}\right)}{4-3 d^{2}} \\
& Q_{1}^{*}=\frac{\left(A\left(1-P_{\text {int }}\right)-C\right)(2-d)}{4-3 d^{2}} \\
& Q_{2}^{*}=\frac{\left(A\left(1-P_{\text {int }}\right)-C\right)\left(2-d-d^{2}\right)}{4-3 d^{2}} \\
& \pi_{1}^{*}=\frac{\left(A\left(1-P_{\text {int }}\right)-C\right)^{2}(2-d)^{2}\left(1-d^{2}\right)}{\left(4-3 d^{2}\right)^{2}} \\
& \pi_{2}^{*}=\frac{\left(A\left(1-P_{\text {int }}\right)-C\right)^{2}\left(2-d-d^{2}\right)^{2}}{\left(4-3 d^{2}\right)^{2}}
\end{aligned}
$$

With perfectly homogeneous products and symmetric cost, stable equilibrium can be obtained. The equilibrium price equals marginal cost and relay 2 produces zero output, while relay 1 produces the perfectly competitive level of output. The presence of a potential Bertrand-type entrant guarantees a competitive outcome and significantly affects the market. When products are differentiated, in order to assure a stable equilibrium , the degree of differentiation must be sufficiently high [15] and relay 1 gets a strategic advantage over relay 2 .

\section{Stakelberg Model}

In the Stakelberg 2-period model, we investigate the dynamic Cournot leader-follower scenario when the choice of strategic variable is quantity [7] and relays move sequentially 
with rest all assumptions same as $\mathrm{CC}$ model. This decision is irreversible and cannot be changed in the second period. The utility function of the $i^{\text {th }}$ relay is

$$
\begin{aligned}
& \pi_{1}=\left(A\left(1-P_{\text {int }}\right)-\left(Q_{1}+Q_{2}\right)\right) Q_{1}-C Q_{1} \\
& \pi_{2}=\left(A\left(1-P_{\text {int }}\right)-\left(Q_{2}+Q_{1}\right)\right) Q_{2}-C Q_{2}
\end{aligned}
$$

The best response $Q_{2}^{*}$ of follower relay is given as

$$
Q_{2}^{*}=\frac{A\left(1-P_{\text {int }}\right)-C-Q_{1}}{2}
$$

The best response $Q_{1}^{*}$ of leader relay, after knowing the best response of the follower relay $Q_{2}^{*}$, is given as

$$
\begin{aligned}
\pi_{1} & =\left(A\left(1-P_{i n t}\right)-\left(Q_{2}^{*}+Q_{1}\right)\right) Q_{1}-C Q_{1} \\
Q_{1}^{*} & =\frac{A\left(1-P_{i n t}\right)-C}{2}
\end{aligned}
$$

After substituting both equations, we get the dynamic Cournot Nash equilibrium solution as

$$
\begin{aligned}
\left(Q_{1}^{*}, Q_{2}^{*}\right) & =\left(\frac{A\left(1-P_{i n t}\right)-C}{2}, \frac{A\left(1-P_{i n t}\right)-C}{4}\right) \\
\left(\pi_{1}^{*}, \pi_{2}^{*}\right) & =\left(\frac{\left(A\left(1-P_{i n t}\right)-C\right)^{2}}{8}, \frac{\left(A\left(1-P_{i n t}\right)-C\right)^{2}}{16}\right)
\end{aligned}
$$

Under this dynamic sequential symmetric Cournot game, leader will always have a strategic advantage over others [7].

\section{E. Monopoly Model}

In this Monopoly model, there is only single relay (monopolist), who decides on quantity $Q$ and trades each unit of power at marginal cost $C$. The market price, $P$ is determined by inverse market demand $Q$.

$$
P_{1}=A\left(1-P_{\text {int }}\right)-Q
$$

The relays profit function, if it produces $Q$ units is

$$
\pi_{1}=\left(A\left(1-P_{\text {int }}\right)-Q\right) Q-C Q
$$

The relay seeks to maximize its profit by choosing $Q$ such that

$$
Q_{1}^{*}=\frac{\left(A\left(1-P_{\text {int }}\right)-C\right)}{2}
$$

The equilibrium price $P_{1}^{*}$ and utility $\pi_{1}^{*}$ is given as

$$
\begin{aligned}
P_{1}^{*} & =\frac{\left(A\left(1-P_{\text {int }}\right)+C\right)}{2} \\
\pi_{1}^{*} & =\frac{\left(A\left(1-P_{\text {int }}\right)-C\right)^{2}}{4}
\end{aligned}
$$

The relay earns high incentives when it has no other competition in the market.

\section{Asymptotic AnALYSis}

When source-relay and or relay-destination link SNRs are increased asymptotically as compared to eavesdropper's link, the behavior of intercept probability becomes important for system design.This can happen if, as compared to the eavesdropper, source-relay and or relay-destination are very closely placed. Asymptotic analysis provides simpler expression to understand the behavior at a limiting case of high SNR as a function of constituent parameters, with the variation of those parameters. In this section, asymptotic analysis is provided for intercept probability of single DF threshold based relay system. Two cases are of main importance, 1) balanced case, where $S-R_{i}$ and $R_{i}-D$ link average SNRs are same, for all $i$, and together tends to infinity, i.e. $1 / \beta_{s r_{i}}=1 / \beta_{r_{i} d}=$ $1 / \beta \rightarrow \infty, 2)$ unbalanced case, where when either of the $S-R_{i}$ or $R_{i}-D$ for all $i$, link average SNR tends to infinity, i.e. $1 / \beta_{s r_{i}}$ is fixed and $1 / \beta_{r_{i} d}=1 / \beta \rightarrow \infty$, or $1 / \beta_{r_{i} d}$ is fixed and $1 / \beta_{s r_{i}}=1 / \beta \rightarrow \infty$.

\section{A. Single Relay: Balanced Case}

For the balanced case, when $1 / \beta_{s r_{i}}=1 / \beta_{r_{i} d}=1 / \beta \rightarrow \infty$, the intercept probability of dual-hop single relay system in (2) can be expressed as

$$
\begin{aligned}
P_{i n t}^{i(A S)} & =\frac{\beta_{r_{i} d}}{\alpha_{r_{i} e}}+\gamma_{t h} \beta_{s r_{i}} \\
& =\beta\left[\frac{1}{\alpha_{r_{i} e}}+\gamma_{t h}\right] \\
& =\frac{1}{\frac{1}{\beta}}\left[\frac{1}{\alpha_{r_{i} e}}+\gamma_{t h}\right]
\end{aligned}
$$

This shows that at a very high main channel $\operatorname{SNR}(1 / \beta)$, intercept probability is inversely proportional to $1 / \beta$ and it tends to zero. It is directly proportional to the eavesdropper channel SNR $\left(1 / \alpha_{r_{i} e}\right)$ and required threshold $\gamma_{t h}$.

Diversity order is an important measure of how fast the intercept probability is decreasing as SNR tends to infinity. It also provides an intuitive understanding into the effect of the number of relays on the intercept probability. The diversity order [19] is defined as

$$
D=-\lim _{\mathrm{SNR} \rightarrow \infty} \frac{\log P_{\text {int }}(\mathrm{SNR})}{\log (\mathrm{SNR})},
$$

where $P_{\text {int }}(\mathrm{SNR})$ is the intercept probability as a function of $\mathrm{SNR}=1 / \beta$. Diversity order of (37) can be obtained as one, using this definition. Diversity order is same as the power of the SNR at the denominator of (37). It is also given by the slope of the curve in log graph. As there is no relay selection, it is intuitive that this single relay system achieves diversity order of one.

\section{B. Single Relay: Unbalanced Case}

For the unbalanced case, the behavior of intercept probability is studied keeping the average SNR of the sourcerelay link fixed and asymptotically increasing the average SNR of the relay-destination link, i.e. when $1 / \beta_{s r_{i}}$ is fixed and $1 / \beta_{r_{i} d}=1 / \beta \rightarrow \infty$.

$$
P_{\text {int }}^{i(A S)}=\left[1-\exp \left(-\gamma_{t h} \beta_{s r_{i}}\right)\right]+\frac{1}{\frac{1}{\beta}}\left[\frac{\exp \left(-\gamma_{t h} \beta_{s r_{i}}\right)}{\alpha_{r_{i} e}}\right]
$$

Also, the behavior of intercept probability is studied keeping the average SNR of the relay-destination link fixed and asymp- 
totically increasing the average SNR of the source-relay link, i.e. when $1 / \beta_{r_{i} d}$ is fixed and $1 / \beta_{s r_{i}}=1 / \beta \rightarrow \infty$.

$$
P_{\text {int }}^{i(A S)}=\left[\frac{\beta_{r_{i} d}}{\beta_{r_{i} d}+\alpha_{r_{i} e}}\right]+\frac{1}{\frac{1}{\beta}}\left[\frac{\gamma_{t h} \alpha_{r_{i} e}}{\beta_{r_{i} d}+\alpha_{r_{i} e}}\right]
$$

The asymptotic intercept probability is expressed as a summation of a constant quantity and an asymptotically varying term with $1 / \beta$. At low SNR asymptotically varying term dominates but at high SNR it vanishes. It can be understood that unbalance is caused due to fixing average SNR of any hop in dual-hop system. Even if average SNR of the other hop is infinitely increased, the intercept probability is limited to a constant.

\section{NUMERICAL ANALYSIS}

This section describe the analytical results with MATLAB simulations of a threshold based dual hop DF cooperative relay network, which exactly match the simulated ones. Fig. 1 shows the intercept probability $P_{\text {int }}$ of single $i^{\text {th }}$ relay, as expressed in (2) for the balanced case with total SNR $1 / \beta$ with main link SNR $1 / \beta_{s r_{i}}=1 / \beta_{r_{i} d}=1 / 2 \beta$, as total power is divided equally among the source and relay, also considering equal noise power at each terminal. The figure is plotted with different relay to eavesdropper average SNR $1 / \alpha_{r_{i} e}=1 / \alpha=3,6,9,12$ $\mathrm{dB}$ and $\gamma_{t h}=3,6 \mathrm{~dB}$. Corresponding asymptotic analysis as expressed in (37) is also shown by solid straight lines passing through the curves. It is observed from the figure that improvement in eavesdropper channel quality increases the intercept probability. Also, intercept probability increases with increase in $\gamma_{t h}$.

In Fig. 2, the intercept probability $P_{\text {int }}$ of single $i^{t h}$ relay, as expressed in (2) is plotted for the unbalanced case with average SNR of $1 / \beta_{s r_{i}}=1 / \beta$ at a given $1 / \beta_{r_{i} d}=1 / \beta_{r d}=25,30,35$ $\mathrm{dB}$ with $1 / \alpha_{r_{i} e}=1 / \alpha=6 \mathrm{~dB}$ and $\gamma_{t h}=3 \mathrm{~dB}$, and it is also plotted for the unbalanced case with average SNR of $1 / \beta_{r_{i} d}=1 / \beta$ at a given $1 / \beta_{s r_{i}}=1 / \beta_{s r}=25,30,35 \mathrm{~dB}$ with $1 / \alpha_{r_{i} e}=1 / \alpha=6 \mathrm{~dB}$ and $\gamma_{t h}=3 \mathrm{~dB}$. It is observed that $P_{\text {int }}$ tends to a fixed constant for a given $1 / \beta_{r d}$ or $1 / \beta_{s r}$, even if $1 / \beta$ increases. We can interpret from this flooring of curves that the intercept probability is constrained by either of the $S-R_{i}$ or $R_{i}-D$ link quality. It is interesting to observe that the asymptotically varying term shown as straight solid line, crosses dashed lines at the point after which average SNR of the hop exceeds the average SNR of the other hop.

In Fig. 3, utility $\pi_{1}$ of single relay 1 is plotted for the Cournot duopoly model as expressed in (13) for balanced case with main link SNR $1 / \beta_{s r_{1}}=1 / \beta_{r_{1} d}=1 / 2 \beta$. The figure is plotted with different relay to eavesdropper average SNR $1 / \alpha_{r_{1} e}=1 / \alpha=3,6,9,12 \mathrm{~dB}$ and $\gamma_{t h}=3,6 \mathrm{~dB}$ with product differentiation parameter $d=1$ as homogeneity is considered, while other constants are taken as $A=19$, assuming cost of production, $C=1$. It is observed from the figure that improvement in eavesdropper channel quality degrades the utility $\pi_{1}$ of relay. Also, increase in $\gamma_{t h}$ lowers the utility of relay, especially at low SNR values. It is also interesting to observe that the utility of relay node increases

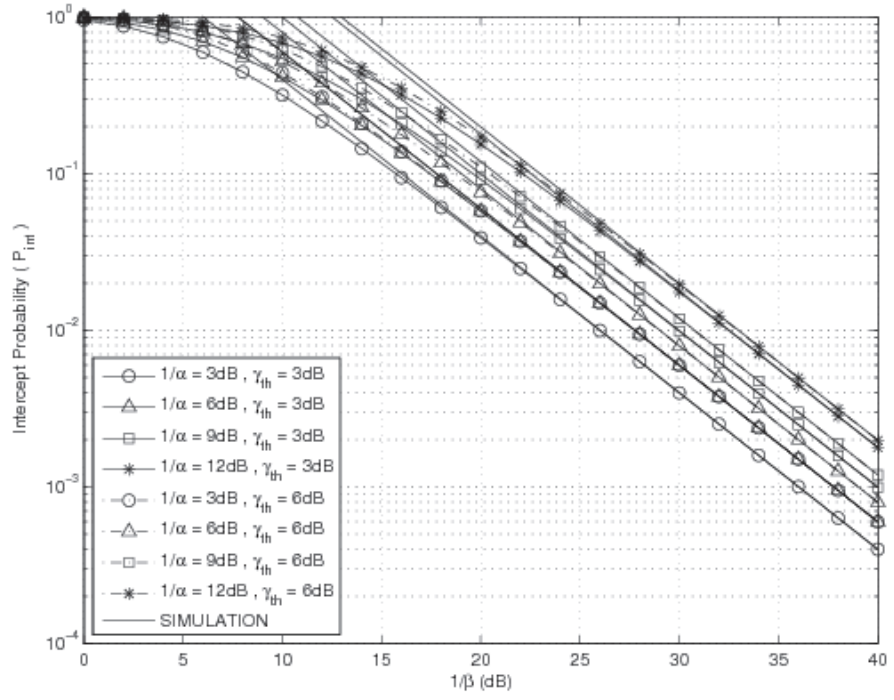

Fig. 1. Intercept probability of single balanced dual hop DF relay system for $1 / \alpha=3,6,9,12 \mathrm{~dB}$ and $\gamma_{t h}=3,6 \mathrm{~dB}$.

with increase in SNR of the main link, especially for lower values of SNR. This observation holds true for other duopoly economic models also.

In Fig. 4, comparison of utility $\pi_{1}$ of single relay 1 for various duopoly economic strategic power trading models is presented for balanced case with main link SNR $1 / \beta_{s r_{1}}=$ $1 / \beta_{r_{1} d}=1 / 2 \beta$. The figure is plotted with different relay to eavesdropper average SNR $1 / \alpha_{r_{1} e}=1 / \alpha=3,6 \mathrm{~dB}$ and $\gamma_{t h}=3 \mathrm{~dB}$ with product differentiation parameter $d=1$ as homogeneity is considered, while other constants are taken as $A=19$ and assuming cost of production, $C=1$. The relay will earn maximum incentives under no competition, which is 2.25 times of the CC model. Under static conditions, with homogeneous goods, relay's dominant strategy is to compete in output rather than price. The profits and price will be more for the relay under $\mathrm{CC}$ scheme, where it chooses output as its strategic variable, as compared to $\mathrm{BB}$ scheme, where relays compete in price. $\mathrm{CB}$ and $\mathrm{BB}$ model guarantees a perfectly competitive equilibrium, where price equals marginal cost $C$. Also, comparing Cournot and Stakelberg model, relay will be better off if the strategy of its opponent is known to him, such that it takes the first mover advantage. Utility of relay under Stakelberg model is $12.5 \%$ greater than Cournot model.

\section{CONCLUSION}

In this paper, intercept probability of a dual hop threshold based cooperative DF relay network for both balanced and unbalanced case with asymptotic analysis is discussed. We observe that improvement in eavesdropper channel quality has a significant impact on the utility of relay especially at low SNR. The relay's profit also increases with increase in SNR of the main link. This motivates relay nodes to maintain low intercept probability to earn high incentives in this market game. We also observe that under comparative analysis of various novel economic duopoly power trading models, the relay has a strategic advantage if it chooses to compete in 


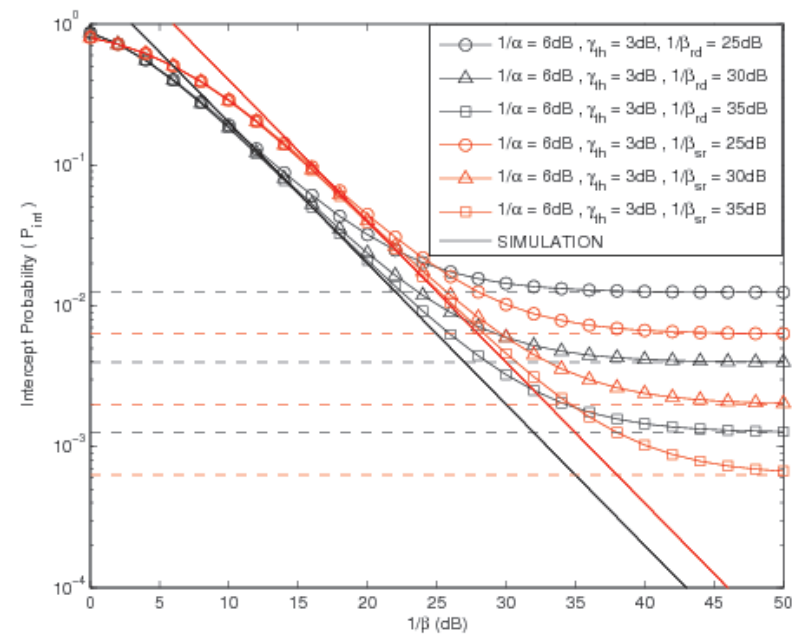

Fig. 2. Intercept probability of single unbalanced dual hop DF relay system for $1 / \alpha=6 \mathrm{~dB}, \gamma_{t h}=3 \mathrm{~dB}$ with $1 / \beta_{r d}=25,30,35 \mathrm{db}$ and $1 / \beta_{s r}=$ $25,30,35 \mathrm{db}$.

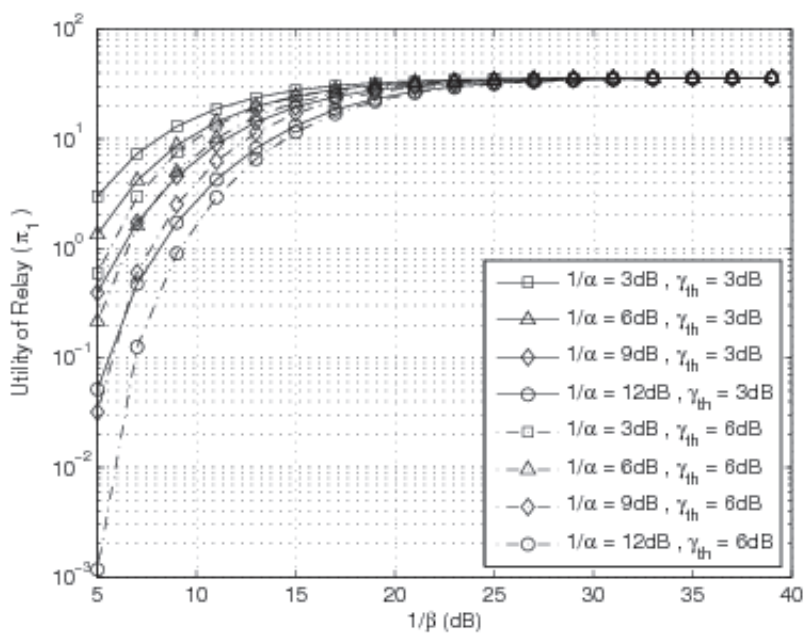

Fig. 3. Utility $\pi_{1}$ of single balanced dual hop DF relay system for $1 / \alpha=$ $3,6,9,12 \mathrm{~dB}$ and $\gamma_{t h}=3,6 \mathrm{~dB}$.

output rather than price in a static and homogeneous scenario. Under dynamic settings, competing in output after observing the opponent's strategy is $12.5 \%$ more profitable for the relay.

\section{REFERENCES}

[1] G. Y. Li, Z. Xu, C. Xiong, C. Yang, S. Zhang, Y. Chen, and S. Xu, "Energy-efficient wireless communications: tutorial, survey, and open issues," Wireless Communications, IEEE, vol. 18, no. 6, pp. 28-35, 2011.

[2] P. Cao and E. Jorswieck, "Source energy-saving performance in amplifyand-forward relay-assisted wireless systems," in Vehicular Technology Conference (VTC Spring), 2013 IEEE 77th. IEEE, 2013, pp. 1-5.

[3] L. Lai and H. E. Gamal, "The relay-eavesdropper channel: Cooperation for secrecy," Information Theory, IEEE Transactions on, vol. 54, no. 9 , pp. 4005-4019, 2008.

[4] L. Dong, Z. Han, A. P. Petropulu, and H. V. Poor, "Improving wireless physical layer security via cooperating relays," Signal Processing, IEEE Transactions on, vol. 58, no. 3, pp. 1875-1888, 2010.

[5] J. Li, A. P. Petropulu, and S. Weber, "On cooperative relaying schemes for wireless physical layer security," Signal Processing, IEEE Transactions on, vol. 59, no. 10, pp. 4985-4997, 2011.

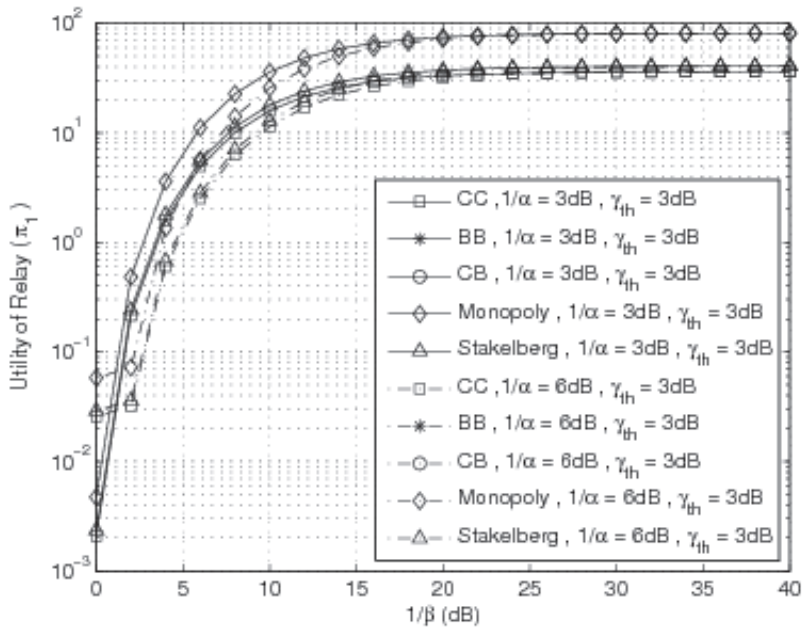

Fig. 4. Comparison of utility $\pi_{1}$ of single balanced dual hop DF relay system for various duopoly economic models with $1 / \alpha=3,6 \mathrm{~dB}$ and $\gamma_{t h}=3 \mathrm{~dB}$

[6] V. N. Q. Bao and N. Trung, "Multihop decode-and-forward relay networks: Secrecy analysis and relay position optimization," Journal on Electronics and Communication (JEC), vol. 2, no. 1-2, pp. 33-42, 2012.

[7] V. J. Tremblay, C. H. Tremblay, and K. Isariyawongse, "Endogenous timing and strategic choice: The cournot-bertrand model," Bulletin of Economic Research, vol. 65, no. 4, pp. 332-342, 2013.

[8] C. H. Tremblay, M. J. Tremblay, and V. J. Tremblay, "A general cournotbertrand model with homogeneous goods," Theoretical Economics Letters, vol. 1 , no. 02 , p. $38,2011$.

[9] U. Nadav and G. Piliouras, "No regret learning in oligopolies: cournot vs. bertrand," in Algorithmic Game Theory. Springer, 2010, pp. 300311

[10] B. Nikfar and S. Maghsudi, "A new bandwidth allocation scheme for dual-hop transmission using virtual oligopoly market model and cournot competition," in Communication Technologies Workshop (Swe-CTW), 2011 IEEE Swedish. IEEE, 2011, pp. 98-102.

[11] M. Dong, X. Liu, Z. Qian, A. Liu, and T. Wang, "Qoe-ensured price competition model for emerging mobile networks," Wireless Communications, IEEE, vol. 22, no. 4, pp. 50-57, 2015.

[12] S. Kandeepan, S. K. Jayaweera, and R. Fedrizzi, "Power-trading in wireless communications: a cooperative networking business model," Wireless Communications, IEEE Transactions on, vol. 11, no. 5, pp. $1872-1880,2012$

[13] A. A. Salem, M. Shokair, M. Elkordy, and S. El Halafawy, "Profit of price with supermodular game for spectrum sharing in cognitive radio using genetic algorithm," Wireless Personal Communications, vol. 82, no. 4, pp. 2601-2609, 2015.

[14] N. Singh and X. Vives, "Price and quantity competition in a differentiated duopoly," The RAND Journal of Economics, pp. 546-554, 1984.

[15] C. H. Tremblay and V. J. Tremblay, "The cournot-bertrand model and the degree of product differentiation," Economics Letters, vol. 111, no. 3, pp. 233-235, 2011

[16] Y. Zou, X. Wang, and W. Shen, "Intercept probability analysis of cooperative wireless networks with best relay selection in the presence of eavesdropping attack," in Communications (ICC), 2013 IEEE International Conference on. IEEE, 2013, pp. 2183-2187.

[17] F. AL-Qahtani, C. Zhong, and H. Alnuweiri, "Opportunistic relay selection for secrecy enhancement in cooperative networks," 2015.

[18] J. Barros and M. R. Rodrigues, "Secrecy capacity of wireless channels," in Information Theory, 2006 IEEE International Symposium on. IEEE, 2006, pp. 356-360.

[19] Y. Zou, X. Wang, and W. Shen, "Optimal relay selection for physicallayer security in cooperative wireless networks," Selected Areas in Communications, IEEE Journal on, vol. 31, no. 10, pp. 2099-2111, 2013. 